\section{Les cellules souches \\ embryonnaires \\ Du développement \\ myocardique \\ à la médecine régénératrice}

\section{Michel Pucéat}

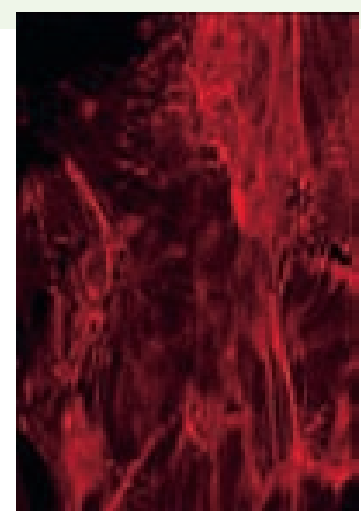

cellules \&S permettent une recherche cognitive sur les réseaux transcriptionnels activés par les morphogènes et qui gouvernent la différenciation cellulaire cardiaque. Bien que les cellules $\varepsilon S$ humaines soient, comme les cellules de souris, isolées à partir du stade blastocyste de l'embryon, des différences dans le développement embryonnaire des deux espèces entraînent des phénotypes cellulaires et des propriétés spécifiques à chaque espèce. Les enjeux actuels sont donc de comprendre les mécanismes moléculaires des processus de spécification et de différenciation cardiaque des cellules $E S$ humaines afin d'accroître leur potentiel cardiogénique. Ce but atteint, ces cellules permettront l'étude du développement cardiaque précoce, nécessaire à une meilleure compréhension des maladies cardiaques congénitales et ouvriront les perspectives de thérapie régénératrice du myocarde. <

Article reçu le 17 mai 2005, accepté le 8 juillet 2005.
CNRS FRE2593, Centre de Recherches de Biochimie macromoléculaire, 1919, route de Mende, 34293 Montpellier Cedex, France.

Les cellules souches michel.puceat@crbm.cnrs.fr embryonnaires :

un espoir thérapeutique?

Bien que les cellules souches soient devenues un thème très à la mode de la biologie du XXI siècle, la recherche sur ces cellules date de plusieurs décennies. En effet, l'intérêt pour ces cellules a commencé à la découverte par Charles Philippe Leblond de cellules, résidant dans des tissus adultes, capables de s'autorenouveler et de se différencier en progéniteurs d'autres types cellulaires [1]. Les cellules souches embryonnaires (ou ES) sont dérivées de l'embryon précoce (stade blastocyste) sous forme de lignées immortelles. Elles ont également la capacité de s'autorenouveler et de se différencier en engendrant les trois feuillets embryonnaires, puis tous les tissus de l'organisme adulte. Cette pluripotence cellulaire est conservée in vitro en culture. Étudiant au laboratoire les mécanismes fondamentaux de la détermination et de la différenciation cardiaques de 
ces cellules, nous observons des foyers contractiles constitués de cellules cardiaques organisées en réseau, une semaine après avoir induit la différenciation au sein de corps embryoïdes, structures tridimensionnelles produites par agrégation des cellules ES et présentant les trois feuillets embryonnaires, ectoderme, endoderme et mésoderme. Ces cellules cardiaques acquièrent différents phénotypes: pacemaker, ventriculaire ou auriculaire (Figure 1) révélant l'extraordinaire potentiel de différenciation de ces cellules. Depuis l'isolement de la première lignée humaine en 1998 [2], ces cellules ont ouvert de vastes perspectives d'étude du développement de l'embryon humain et ont apporté de grands espoirs de thérapie cellulaire de maladies dégénératives dont l'insuffisance cardiaque.

Nadial Rosenthal, chercheur en myologie, a récemment écrit, dans une revue traitant des thérapies cellulaires potentielles, un scénario de science fiction sur lequel de nombreux laboratoires travaillent de par le monde: un patient souffrant de défaillance cardiaque reçoit une transplantation de cellules souches embryonnaires personnalisées grâce au transfert nucléaire. Les cellules trouvent un environnement de facteurs de croissance favorable et se différencient en cardiomyocytes régénérant le myocarde (Figure 2) [3]. Ce rêve deviendra-t-il un jour prochain une réalité pour les six cents millions de personnes à haut risque de déficience cardiaque vivant sur notre planète?

\section{Potentiel cardiogénique des cellules souches embryonnaires murines}

La première lignée de cellules souches murines a été isolée il y a vingtaine d'années [4]. Une publication d'un groupe allemand, pionner dans le domaine, a

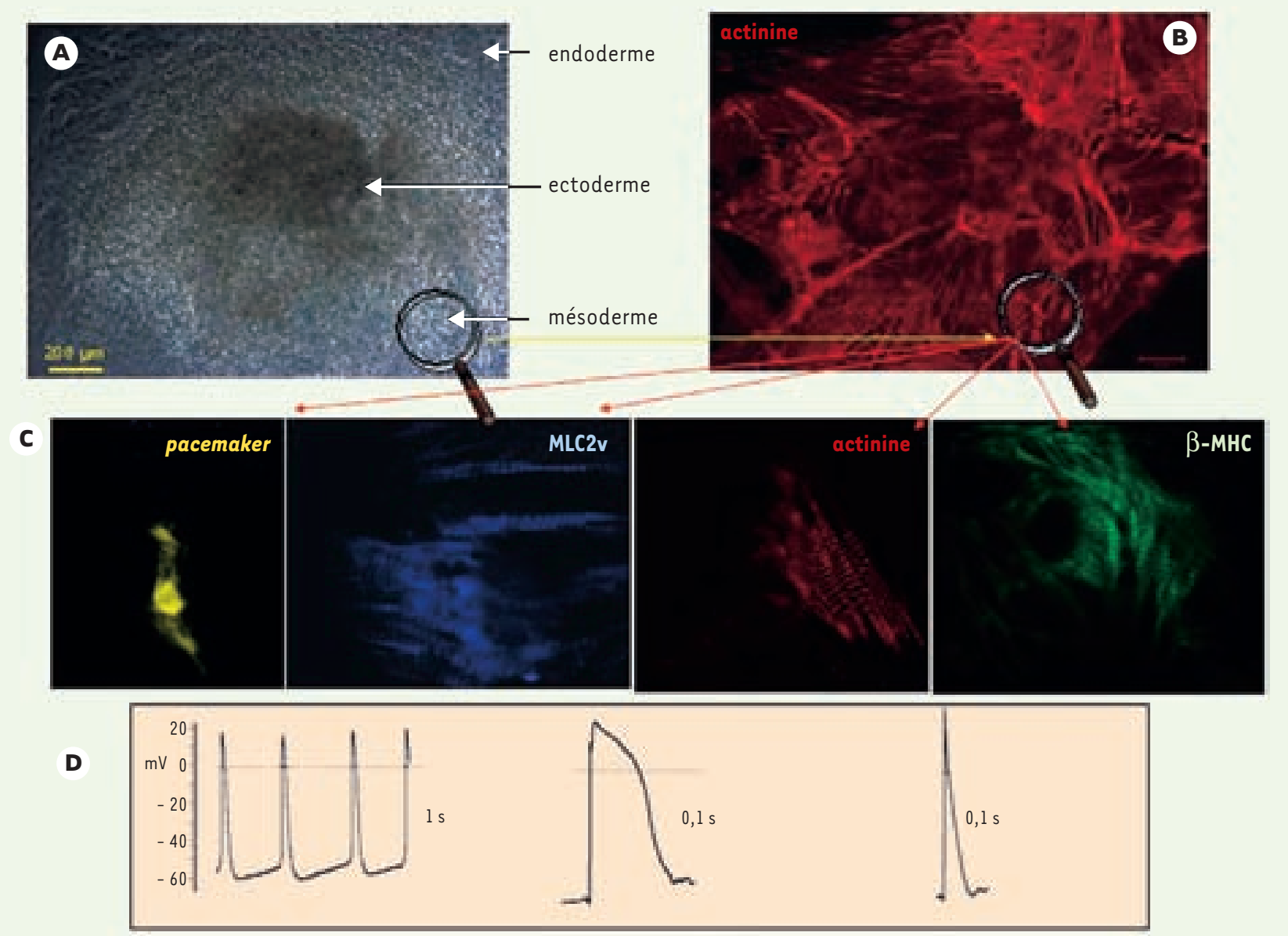

Figure 1. Multipotentialité cardiogénique des cellules ES. A. Formation de corps embryoïdes. B. Structure tridimensionnelle (incluant les trois feuillets embryonnaires à partir de cellules $\varepsilon S$ ) donnant naissance au sein du mésoderme à des cellules cardiaques organisées en réseau de cellules exprimant la protéine sarcomérique actinine. C. Ces réseaux comprennent des cellules pacemaker, ventriculaires (exprimant la chaîne légère 2 de myosine ventriculaire) et atriales. $\boldsymbol{D}$. Ces cellules développent des potentiels d'action spontanés et courts (pacemaker) ou induits et longs (ventriculaire) ou induits et courts (auriculaire). 
montré il y a une dizaine d'années que les cellules ES murines se différenciaient en différent types cellulaires rencontrés dans le myocarde: les cellules pacemaker et de conduction, les cellules auriculaires et ventriculaires [5]. Nous avons étudié plus en détail cette multipotentialité cardiogénique des cellules ES de souris. Utilisant des protéines fluorescentes dont l'expression dans les cellules ES est pilotée par des promoteurs cardiaques, nous avons pu suivre la destinée des cellules et caractériser leur phénotype. Nous avons pu confirmer la présence de cellules auriculaires et ventriculaires [6] dans les corps embryoïdes, ainsi que des cellules pacemaker [7] (Figure 1).

Il est possible de favoriser la différenciation cardiaque ventriculaire en traitant les cellules par des facteurs cardiogéniques de la superfamille du TGF $\beta$ (transforming growth factor $\beta$ ), sécrété par l'embryon au stade prégastrulation [8], puis par l'endoderme. Les cellules expriment alors des gènes spécifiques du mésoderme puis du myocarde [9]. Le BMP2 (bone morphogenic protein) est l'un des membres de cette superfamille dont la fonction cardiogénique est la mieux conservée au cours de l'évolution des organismes depuis Dpp, son homologue chez la drosophile [10], jusque chez le poulet [11], en passant par le poisson zèbre (swr/bmp2b) [12] ou le xénope [13]. Cette réponse transcriptionnelle au TGF $\beta$ ou BMP2 valide les cellules $\varepsilon S$ comme modèle de développement cardiaque précoce. II reste pourtant à déterminer si ces cellules peuvent engendrer les deux champs ou lignages cardiaques, récemment mis en en évidence [14]. En effet, le champ primaire, au sein duquel les cellules expriment notamment Nkx2.5, Mef2c, GATA et Tbx5, donne naissance au ventricule gauche et aux oreillettes, alors que le ventricule droit et le tronc artériel émanent du champ secondaire [14], caractérisé en particulier par des cellules progénitrices exprimant les facteurs Is|l, Tbxl et FoxHl [15-17]. S'il est bien établi que les cellules $\varepsilon S$ engendrent le champ primaire in vitro, il reste à démontrer qu'elles produisent aussi le champ secondaire.

La cicatrice fibreuse qui se développe dans le myocarde après un épisode ischémique secrète des facteurs de la superfamille du TGF $\beta$ [18]. Cette sécrétion paracrine peut donc être utilisée en thérapie régéné-

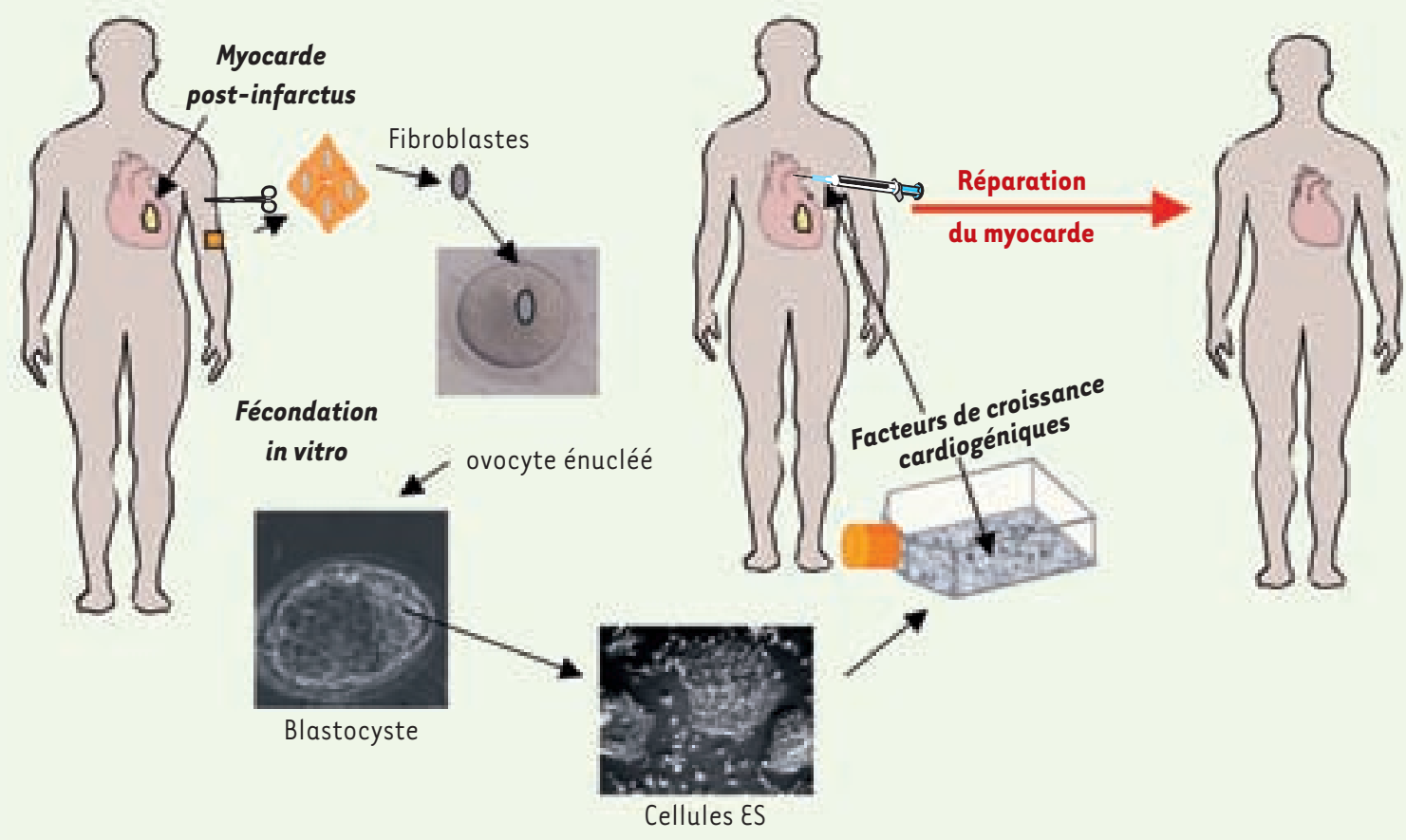

Figure 2. Transfert nucléaire somatique et réparation myocardique : un scénario futuriste. Des fibroblastes prélevés dans la peau d'un patient fournissent le noyau somatique transplanté dans un ovocyte énucléé. Après fécondation artificielle, l'ovocyte se développe par division cellulaire en blastocyste à partir duquel sont isolées des cellules ES. Ces dernières sont spécifiées vers le phénotype cardiaque avec des facteurs de croissance, et sont transplantées dans le myocarde. Trouvant les mêmes facteurs cardiogéniques in situ, les cellules se différencient en cardiomyocytes et régénèrent le myocarde. 
ratrice car ce processus permet en effet la différenciation de cellules ¿S murines vers un phénotype cardiaque après greffe intramyocardique chez le rat [9-19] ou chez le mouton [20], régénérant le myocarde et améliorant sa contractilité. II faut mentionner dans ces expériences l'absence de tératomes chez ces animaux [21], indiquant que la réponse des cellules compétentes non différenciées aux morphogènes inhibe tout phénomène d'hyperprolifération. Cette situation est assez comparable à celle des greffes de tissus embryonnaires qui perdent leur propriété tératogène très tôt après la différenciation des cellules de l'épiblaste [22], concept qui a été confirmé plus récemment [23]. Toutes ces données nous rapprochent de la réalisation du scénario de science fiction. II reste néanmoins encore deux inconnues majeures pour rendre ce scénario réalisable : le potentiel cardiogénique réel des cellules ES humaines et la tolérance immunologique des cellules ES.

\section{Potentiel cardiogénique des cellules souches embryonnaires humaines}

Kehat et al. [24] furent les premiers auteurs à montrer la différenciation des cellules $E S$ humaines en cardiomyocytes. Ces cellules ne se différencient pas néanmoins jusqu'au stade terminal. De plus, elles ne représentent qu'une faible population cellulaire au sein des corps embryoïdes. En 2003, une étude électrophysiologique [25] a pourtant démontré la présence de cellules auriculaires, ventriculaires et nodales parmi les cardiomyocytes dérivés des cellules \&S humaines. Cependant, la plupart des cardiomyocytes ont un phénotype précoce à activité électrique spontané, une propriété des premiers stades de différenciation cardiaque. Bien que cette propriété ait été utilisée pour engendrer un pacemaker biologique $[26,27]$, cet état n'est que transitoire, les cellules devant ensuite devenir quiescentes. La différenciation des cellules humaines en cardiomyocytes n'est pas aussi spontanée que celle des cellules de souris. Des facteurs extrinsèques sont donc nécessaires. Les cellules $\varepsilon S$ expriment les récepteurs membranaires pour les facteurs de la superfamille du TGF $\beta$, et la famille des FGF (fibroblast growth factor) [28]. Le TGF $\beta$ et l'activine orientent la différenciation des cellules $E S$ humaines vers un lignage du mésoderme [29]. Nous avons pu observer une induction de gènes cardiaques (GATA4, Mef2c et $N k \times 2.5$ ) après stimulation de cellules $E S$ de singe Rhésus par le BMP2. Des cellules endodermiques ( $E N D-2)$ qui sécrètent des facteurs cardiogéniques, dont le TGF $\beta$, les FGF et le BMP2 facilitent aussi l'orientation cardiogénique des cellules humaines [30]. Ces données préliminaires montrent donc que les cellules humaines suivent un programme génétique de différenciation assez similaire sans doute à ce qui se passe chez l'embryon in vivo, mais de nombreux travaux restent nécessaires pour réussir à accroître le potentiel cardiogénique de ces cellules.

\section{Privilège immun des cellules souches embryonnaires}

Nos observations de la tolérance des cellules ES murines chez le rat ou le mouton immunocompétent [9] suggèrent que ces cellules possèdent un privilège immun [31]. Différentes hypothèses pourraient expliquer ce privilège. Les cellules $\varepsilon S$ humaines n'expriment que peu d'antigène d'histocompatibilité et ne sont pas la cible des lymphocytes T [32]. Elles pourraient sécréter un facteur immunosuppresseur local, une propriété des cellules souches mésenchymateuses [33]. Elles pourraient également induire un phénomène de tolérance [34-36]. Leur statut embryonnaire pourrait aussi leur conférer une neutralité immunologique et des facultés d'adaptation à l'environnement du tissu hôte. Tous ces mécanismes doivent être considérés et étudiés afin de caractériser le statut immunologique des cellules ES. De ce dernier dépend en effet la nécessité de manipuler génétiquement les cellules pour neutraliser les antigènes d'histocompatibilité par exemple, ou d'utiliser le transfert nucléaire somatique afin de personnaliser le statut immunologique de la cellule ES (Figure 2).

\section{Facteurs cardiogéniques et réseaux transcriptionnels déterminant le phénotype cardiaque chez l'embryon}

Un des défis actuels de la recherche en développement cardiovasculaire est la quête de l'origine des progéniteurs cardiaques. Cette recherche doit servir de base à toute approche de différenciation des cellules ES en cardiomyocytes. Des expériences de cartographie cellulaire chez l'embryon de souris ont montré que les premiers progéniteurs cardiaques se localisent dans la région latérale postérieure de l'épiblaste juste avant la gastrulation. Au cours de la gastrulation, ces cellules rejoignent le mésoderme en migrant à travers la ligne primitive [37]. Les cellules alors compétentes se trouvent en contact avec l'endoderme, sécrétant des facteurs inducteurs de la cardiogenèse ou morphogènes. Ce processus se déroule en 6 jours chez la souris et en une vingtaine de jours chez l'homme.

Un certain nombre de facteurs de transcription - molécules se liant spécifiquement sur des séquences consensus d'ADN des promoteurs de gènes pour induire leur expression - jouent un rôle important dans le processus de différenciation cellulaire cardiaque in vivo et in vitro dans les corps embryoïdes engendrés à partir des cellules ES [38]. Ces facteurs appartiennent à différentes familles de gènes, les homéoprotéines $N k \times 2$, la famille MADS-box (myocyte enhancer factor-2, Mef2), les facteurs de transcription en doigt de zinc (famille des GATA) et de la T-box, Tbx. Ces facteurs constituent un réseau transcriptionel finement régulé par les facteurs de croissance et de différenciation cellulaire. Les facteurs de transcription cardiaques sont plus particulièrement Nkx2.5, Mef2c, GATA4,5,6, la myocardine, Tbx 5 et Tbx20. Ces facteurs travaillent de concert pour 
induire l'activité transcriptionnelle de promoteurs de gènes cardiaques [39]. Ils sont mis en jeu suite à la stimulation des cellules par des facteurs de croissance ou «morphogènes » qui activent une cascade d'événements intracellulaires (souvent une suite d'activation de kinases) aboutissant à l'activation du facteur de transcription, par exemple en le transloquant dans le noyau de la cellule où il exercera son action. Ces morphogènes appartiennent essentiellement à la superfamille du TGF $\beta$ incluant l'activine, nodal, les BMP ou le TGF $\beta$, ainsi qu'à la famille des FGF. Des signaux inhibiteurs de la différenciation cardiaque et médiés par les glycoprotéines sécrétées Wnt sont réprimés par des antagonistes tels que les molécules Crescent et Dkkl (Dickkopf), ces derniers favorisant donc également la différenciation cardiaque. Wnt 11 est le seul membre de la famille des Wnt qui est cardiogénique [40]. D'autres facteurs tels que Sonic et Indian Hedgehog (Shh et Ihh) favorisent la différenciation cellulaire cardiaque, en étant une cible préférentielle de la signalisation intracellulaire du récepteur FGF [41] et des activateurs directs de l'expression de Nkx2.5 [42]. Plus récemment, la molécule réceptrice Notch-1 et son ligand Serrate, exprimés à la même période que Nkx2.5 dans les territoires précurseurs cardiogéniques de l'embryon, ont été impliqués dans le processus de décision phénotypique cardiaque. Ils seraient des inhibiteurs de ce processus [43]. Tous les gènes codant pour ces facteurs de transcription sont présents dans le génome humain [44]. Les cascades transcriptionelles restent néanmoins encore spéculatives chez l'homme.

La voie des BMP, agissant par l'intermédiaire des facteurs intracellulaires smad est décrite comme la voie cardiogénique royale aussi bien in vivo [45] qu'in vitro à partir de cellules ES [46]. Nous avons récemment mis en évidence une nouvelle voie, indépendante et plus précoce que celle des $B M P$, en étudiant la différenciation des cellules $\varepsilon S$ déficientes en protéine du rétinoblastome $(\mathrm{Rb})$. Les cellules $\varepsilon S R b^{-/-}$se différencient en cardiomyocytes, mais avec un retard significatif par rapport aux cellules ES sauvages. Les facteurs de transcription spécifiques du mésoderme cardiaque (Mesp, Tbx6) et les facteurs cardiaques (Nkx2.5, MEF2C, GATA...) sont exprimés dans les corps embryoïdes de cellules $\varepsilon S R b^{-/-} 5$ jours plus tard que dans les cellules sauvages. La myofribrillogenèse et l'activité contractile sont elles aussi retardées. Ces données suggèrent que $\mathrm{Rb}$ est nécessaire à une étape précoce de différenciation. $\mathrm{Rb}$ a un site de liaison à un facteur cardiogénique aviaire CMFl [47]. Nous avons testé l'hypothèse selon laquelle Rb serait nécessaire à la voie de différenciation impliquant LEKl (homologue de CMFl aviaire chez la souris) en amont, mais indépendante de la voie BMP. En exprimant des ARN interférentiels, et des ADNc antisens ciblant LEKl, ou en inhibant I'interaction Rb/LEKl dans les cellules ES par une séquence peptidique mimant le site de liaison de Rb sur LEKl ciblée dans le noyau, nous avons proposé une nouvelle voie transcriptionnelle cardiogénique indépendante de la voie canonique BMP, mais dépendante d'une interaction $\mathrm{Rb} / \mathrm{LEKl}$ [38] (Figure 3). Cette voie transcriptionnelle est en cours d'étude au laboratoire. La fonctionnalité de ces voies cardiogéniques chez l'homme reste une question à élucider.

\section{Différences entre le développement précoce du myocarde murin et humain}

Les cellules ES humaines sont isolées comme les cellules de souris à partir du stade blastocyste de l'embryon. Il est important de noter néanmoins que le blastocyste humain est plus précoce que celui de la souris, se formant après un stade de division supplémentaire. L'acti-

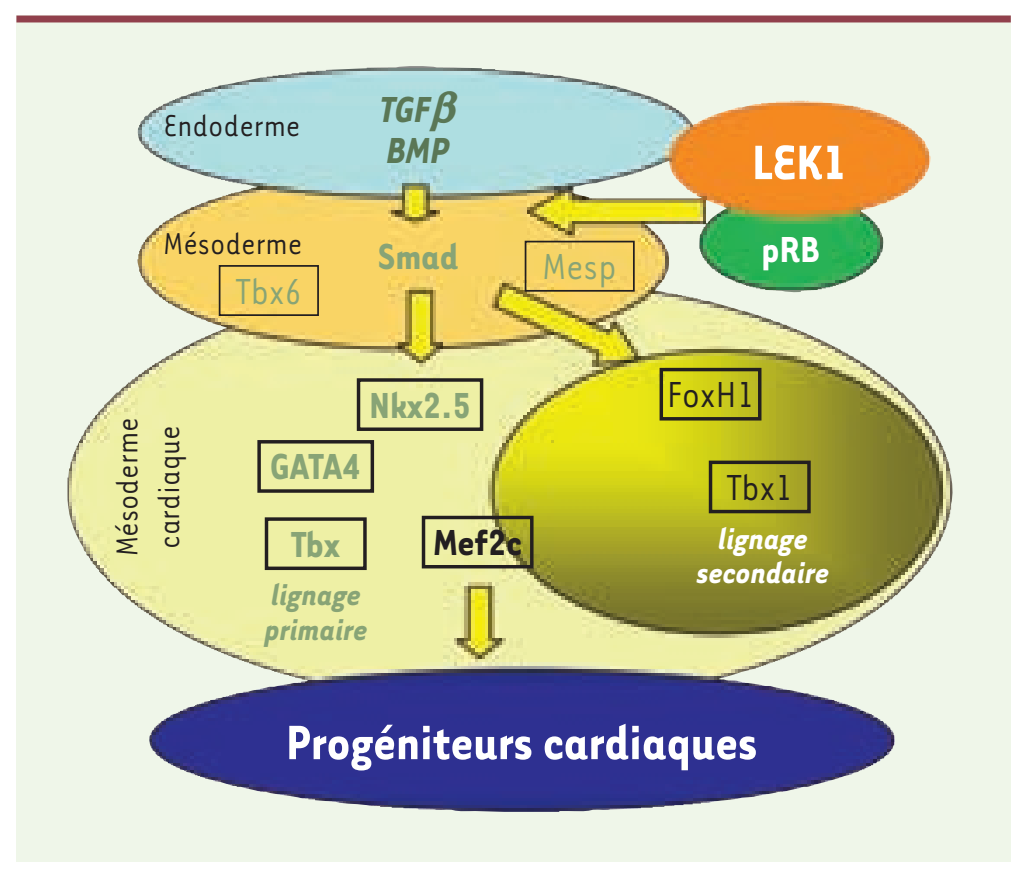

Figure 3. Réseaux transcriptionnels déterminant le phénotype cardiaque in vitro et in vivo. Les progéniteurs cardiaques de l'épiblaste rejoignent, au cours de la gastrulation, le mésoderme en migrant à travers la ligne primitive. Les cellules alors compétentes se trouvent en contact avec l'endoderme sécrétant les facteurs de la superfamille du TGF $\beta$ (TGF $\beta$, BMP2). Ces facteurs activent l'expression de facteurs de transcription. Ces derniers, agissant en combinaison les uns avec les autres, permettent la spécification du mésoderme cardiaque, puis des lignages cardiaques primaire et secondaire. Les cellules du champ primaire donneront naissance aux oreillettes et au ventricule gauche, et celles du champ secondaire au ventricule droit et au tronc artériel. 
vation majeure du génome se produit au stade 2 cellules chez la souris et à 6-8 cellules chez l'homme [48]. Les trois feuillets embryonnaires se forment 6 jours après la fécondation chez la souris et 14 jours après chez l'homme. Les cellules ES humaines sont donc probablement plus précoces dans le stade de développement que les cellules de souris. Ce statut se reflète d'ailleurs au niveau du profil d'expression génique des cellules ES non différenciées ou en cours de différenciation au sein de corps embryoïdes. Les cellules ES non différenciées de souris expriment déjà des marqueurs de l'endoderme viscéral ( $\alpha$-fœto-protéine) et du mésoderme (brachyury). Elles perdent en 5 jours l'expression d'0ct-4, un marqueur de pluripotence, au cours de leur différenciation. Les cellules de primates n'expriment pas ces marqueurs à l'état indifférencié (nos résultats). L' $\alpha$-fœto-protéine n'est détectée qu'aux jours 10-30 dans les corps embryoïdes humains. L'expression d'0ct-4 disparaît dès le cinquième jour dans les corps embryoïdes engendrés à partir de cellules murines alors qu'il persiste au jour 10 dans les corps embryoïdes humains [49]. La pluripotence des cellules de primates a été montrée par formation de tératomes chez les souris immunodéprimées. Néanmoins, aucune formation d'embryons chimériques n'a été réalisée à partir des cellules de primates non humains.

Les mécanismes du maintien de la pluripotence des cellules \&S humaines font également apparaître quelques différences avec ceux qui gouvernent la pluripotence des cellules murines. Notamment les cellules humaines n'ont pas besoin de LIF (leukemia inhibitory factor) comme les cellules de souris. Le LIF est nécessaire au maintien de la pluripotence des cellules souches dans l'embryon de souris en diapause, un état de repos du blastocyste qui n'a pas d'équivalent chez l'homme. Les facteurs de transcription, dont l'expression maintient l'état de pluripotence des cellules de souris ( 0 ct-4 et Nanog), jouent néanmoins le même rôle dans les cellules \&S humaines, témoignant de la conservation de certains mécanismes d'autorenouvellement [50]. Ces différences doivent êtres prises en compte dans l'établissement de stratégies de spécification et de différenciation des cellules $\varepsilon S$ humaines.

\section{Conclusions}

Les travaux présentés dans cette étude démontrent que les cellules ¿S suivent les mêmes routes de spécification et de différenciation tissulaires in vitro qu'au cours de l'embryogenèse. Ces cellules représentent donc un bon modèle de développement précoce du myocarde. Les cellules $E S$ dérivées de blastocystes porteurs de mutations ou d'anomalies génétiques apportent donc un outil précieux d'étude de maladies cardiaques congénitales et génétiques. Les différences de développement des embryons des souris et humains sont sans aucun doute à considérer dans les études de spécification et de différenciation des cellules $E S$ murines et humaines vers le phénotype cardiaque. Une étude documentée des réseaux transcriptionnels déterminant le phénotype cardiaque humain permettra de comprendre les mécanismes de différenciation cellulaire cardiaque et d'envisager la réalisation du scénario de réparation du myocarde de patients souffrant de défaillance cardiaque. $\diamond$

\section{SUMMARY}

Embryonic stem cells to study early myocardial development and potential regenerative medicine Embryonic stem cells are capable to recapitulate the first stages of myocardial development. Using mouse embryonic stem cells, transcriptional networks specifying the cardiac fate can be delineated. Furthermore, using members of the TGF $\beta$ superfamily to commit mouse ES cells toward a cardiac lineage, recent studies showed that ESC-derived cardiomyocytes were capable to repair postinfarcted myocardium of small and large animals. The next challenges are to validate such results using human ESCs in order to better comprehend cardiac congenital diseases and to foresee a cell therapy of heart failure. $\diamond$

\section{REMERCIEMENTS}

L'auteur remercie les étudiants de son équipe qui ont effectué les travaux rapportés dans cette revue, les Drs Atta Behfar, Annabelle Méry, Evangélia Papadimou, Marietta Barro, Dana Zeinnedine, Corinne Grey, Claudine Ménard et Franck Aimond, le Dr Pierre Charnet (CNRS FRE2593, Montpellier) pour les tracés d'électrophysiologie, ainsi que ses collaborateurs cliniciens, les Prs Philippe Menasché et Albert Hagège (HEGP, Paris) et leurs collaborateurs ainsi que ses collaborateurs à la Mayo Clinic (Rochester, USA), dans le laboratoire du Dr André Terzic. Notre recherche est financée par la Fondation de France, l'AFM, la Fondation pour la recherche médicale et l'Inserm.

\section{RÉFÉRENCES}

1. Leblond CP, Walker BE. Renewal of cell populations. Physiol Rev 1956 ; $36: 255-76$.

2. Thomson JA, Itskovitz-Eldor J, Shapiro SS, et al. Embryonic stem cell lines derived from human blastocysts. Science 1998; 282 : 1145-7.

3. Rosenthal N. Youthful prospects for human stem-cell therapy. $\varepsilon M B O$ Rep $2005 ; 6:$ S30-4.

4. Magnuson T, Epstein CJ, Silver LM, et al. Pluripotent embryonic stem cell lines can be derived from tw5/tw5 blastocysts. Nature $1982 ; 298: 750-3$.

5. Maltsev VA, Wobus AM, Rohwedel J, et al. Cardiomyocytes differentiated in vitro from embryonic stem cells developmentally express cardiacspecific genes and ionic currents. Circ Res 1994 ; 75 : 233-44.

6. Meyer N, Jaconi M, Ladopoulou A, et al. A fluorescent reporter gene as a marker for ventricular specification in ES-derived cardiac cells. FEBS Lett $2000 ; 478: 151-8$.

7. Mery A, Aimond F, Menard, et al. Initiation of embryonic cardiac pacemaker activity by inositol 1,4,5 trisphosphate-dependent calcium signaling. Mol Biol Cell $2005 ; 9$ : 2414-23.

8. Rizzino A. Early mouse embryos produce and release factors with transforming growth factor activity. In Vitro Cell Dev Biol $1985 ; 21: 531-$ 6.

9. Behfar A, Zingman L, Hodgson D, et al. Stem cell differentiation requires a paracrine pathway in the heart. FASEB J 2002 ; $16: 1558-66$.

10. Frasch $M$. Intersecting signalling and transcriptional pathways in Drosophila heart specification. Semin Cell Dev Biol 1999; 10:61-71.

11. Andree B, Duprez D, Vorbusch B, et al. BMP-2 induces ectopic expression of cardiac lineage markers and interferes with somite formation in chicken embryos. Mech Dev $1998 ; 70: 119-31$.

12. Reiter JF, Verkade H, Stainier DY. Bmp2b and Oep promote early myocardial differentiation through their regulation of gata5. Dev Biol $2001 ; 234: 330-8$. 
13. Shi $Y$, Katsev $S$, Cai $C$, Evans $S$. BMP signaling is required for heart formation in vertebrates. Dev Biol $2000 ; 224: 226-37$.

14. Kelly RG. Molecular inroads into the anterior heart field. Trends Cardiovasc Med $2005 ; 15: 51-6$.

15. Cai CL, Liang X, Shi Y, et al. Isll identifies a cardiac progenitor population that proliferates prior to differentiation and contributes a majority of cells to the heart. Dev Cell $2003 ; 5: 877-89$.

16. Von Both I, Silvestri C, Erdemir T, et al. Foxhl is essential for development of the anterior heart field. Dev Cell $2004 ; 7: 331-45$.

17. Meilhac SM, Esner M, Kelly RG, et al. The clonal origin of myocardial cells in different regions of the embryonic mouse heart. Dev Cell $2004 ; 6: 685-98$.

18. Sun Y, Weber KT. Infarct scar: a dynamic tissue. Cardiovasc Res $2000 ; 46: 250-6$.

19. Kofidis T, de Bruin JL, Yamane T, et al. Stimulation of paracrine pathways with growth factors enhances embryonic stem cell engraftment and host-specific differentiation in the heart after ischemic myocardial injury. Circulation $2005 ; 111: 2486-93$.

20. Ménard C, Hagège A, Agbulut 0 , et al. Transplantation of mouse cardiaccommitted embryonic stem cells in infarcted sheep myocardium: a preclinical study. Lancet 2006 (sous presse).

21. Behfar A, Hodgson DM, Zingman LV, et al. Administration of allogenic stem cells dosed to secure cardiogenesis and sustained infarct repair. Ann NY Acad Sci 2005 ; 1049: 189-98.

22. Damajanov I, Solter D, Skreb N. Teratocarcinogenesis as related to the age of embryos grafted under the kidney capsule. Roux Arch Dev Biol 1971 ; 173 : 228-34

23. Eventov-Friedman $S$, Katchman $H$, Shezen $\varepsilon$, et al. Embryonic pig liver, pancreas, and lung as a source for transplantation: optimal organogenesis without teratoma depends on distinct time windows. Proc Natl Acad Sci USA 2005 ; 102 : 2928-33.

24. Kehat I, Kenyagin-Karsenti D, Snir M, et al. Human embryonic stem cells can differentiate into myocytes with structural and functional properties of cardiomyocytes. J Clin Invest 2001 ; $108: 407-14$.

25. He Je, Ma Y, Lee Y, et al. Human embryonic stem cells develop into multiple types of cardiac myocytes: action potential characterization. Circ Res 2003; $93: 32-9$.

26. Kehat I, Khimovich L, Caspi 0 , et al. Electromechanical integration of cardiomyocytes derived from human embryonic stem cells. Nat Biotechnol $2004 ; 22: 1282-9$.

27. Xue $\mathrm{T}$, Cho HC, Akar FG, et al. Functional integration of electrically active cardiac derivatives from genetically engineered human embryonic stem cells with quiescent recipient ventricular cardiomyocytes: insights into the development of cell-based pacemakers. Circulation $2005 ; 111: 11-20$.

28. Pera MF, Trounson A0. Human embryonic stem cells: prospects for development. Development $2004 ; 131: 5515-25$.

29. Schuldiner M, Yanuka 0, Itskovitz-Eldor J, et al. Effects of eight growth factors on the differentiation of cells derived from human embryonic stem cells. Proc Natl Acad Sci USA $2000 ; 97$ : 11307-12.

30. Mummery C, Ward-Van Oostwaard D, Doevendans P, et al. Differentiation of human embryonic stem cells to cardiomyocytes: role of coculture with visceral endodermlike cells. Circulation 2003; $107: 2733-40$.

31. Chiu RC. Xenogeneic cell transplant: fact or fancy? Int J Cardiol 2004 ; 95 (suppl 1) : S43-4.

32. Drukker M, Benvenisty $N$. The immunogenicity of human embryonic stem-derived cells. Trends Biotechnol $2004 ; 22: 136-41$.
33. Aggarwal S, Pittenger MF. Human mesenchymal stem cells modulate allogeneic immune cell responses. Blood $2005 ; 105$ : 1815-22.

34. Frandrich F, Lin X, Chai GX, et al. Preimplantation-stage stem cells induce long term allogenic graft acceptance without supplementary host conditioning. Nat Med $2002 ; 8: 171-8$.

35. Li L, Baroja ML, Majumdar A, Chadwick K, et al. Human embryonic stem cells possess immune-privileged properties. Stem Cells $2004 ; 22: 448-56$.

36. Fabricius D, Bonde S, Zavazava N. Induction of stable mixed chimerism by embryonic stem cells requires functional Fas/FasL engagement. Transplantation $2005 ; 79: 1040-4$.

37. Tam PLL, Zhou SX. The allocation of epiblats cells to ectodermal and germ-line lineages is influenced by the position of the cells in the gastrulating mouse embryo. Dev Biol 1996; $178: 124-32$.

38. Papadimou E, Menard C, Grey C, Puceat M. Interplay between the retinoblastoma protein and LEKI specifies stem cells toward the cardiac lineage. EMBOJ $2005 ; 24$ : 1750-61.

39. Harvey RP. Patterning the vertebrate heart. Nat Rev Genet 2002 ; 3 : 544-56.

40. Eisenberg CA, Eisenberg LM. WNTll promotes cardiac tissue formation of early mesoderm. Dev Dyn 1999; $216: 45-58$

41. Dell'Era P, Ronca R, Coco L, et al. Fibroblast growth factor receptor- 1 is essential for in vitro cardiomyocyte development. Circ Res 2003; $93: 414-20$.

42. Zhang XM, Ramalho-Santos M, McMahon AP. Smoothened mutants reveal redundant roles for Shh and Ihh signaling including regulation of L/R asymmetry by the mouse node. Cell $2001 ; 105$ : 781-92.

43. Schroeder T, Fraser ST, Ogawa M, et al. Recombination signal sequencebinding protein J-kappa alters mesodermal cell fate decisions by suppressing cardiomyogenesis. Proc Natl Acad Sci USA $2003 ; 100: 4018-23$.

44. Ryan K, Chin AJ. T-box genes and cardiac development. Birth Defects Res C Embryo Today $2003 ; 69: 25-37$.

45. Olson $\varepsilon N$. Development. The path to the heart and the road not taken. Science $2001 ; 291: 2327-8$.

46. Loebel DA, Watson CM, De Young RA, et al. Lineage choice and differentiation in mouse embryos and embryonic stem cells. Dev Biol $2003 ; 264$ : 1-14.

47. Wei $Y$, Bader D, Litvin J. Identification of a novel cardiac-specific transcript critical for cardiac myocyte differentiation. Development 1996 ; 122 : 2779-89.

48. Latham KE. Mechanisms and control of embryonic genome activation in mammalian embryos. Int Rev Cytol 1999; 193 : 71-124.

49. Dvash T, Mayshar $Y$, Darr H, et al. Temporal gene expression during differentiation of human embryonic stem cells and embryoid bodies. Hum Reprod 2004; $19: 2875$ 83.

50. Rao M. Conserved and divergent paths that regulate self-renewal in mouse and human embryonic stem cells. Dev Biol $2004 ; 275: 269-86$.

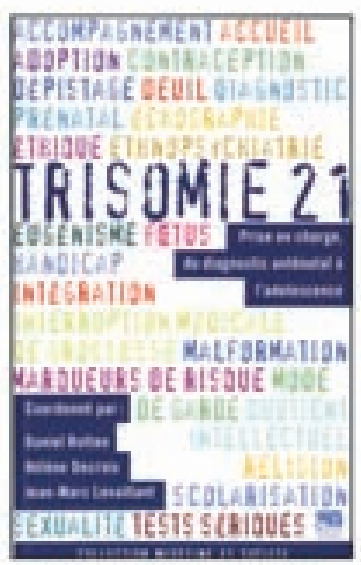

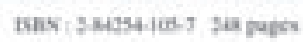

\section{Bon de commande}

A retourner a EDK, Io Villa d'Orléans - 75014 PARIS

Tél. ; 0153910606 - Fax : 0153910607 - E-mail ; cditoriale edk.fr

NOM:

Prénom

Adresse

Code postal

Ville :

Pays

Fosction:

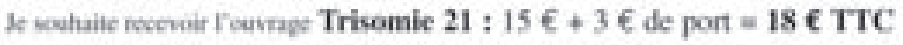

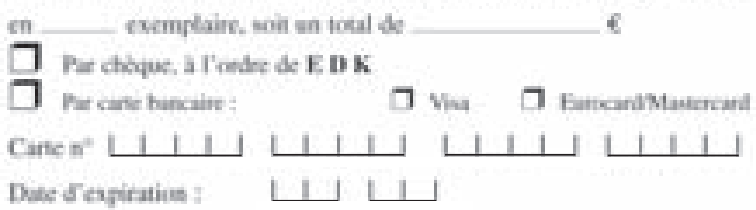

Sivnature
TIRÉS À PART

M. Pucéat 